\section{Editor-in-Chief \\ Barbara McLain - (retired Prof.) \\ University of Hawaii, USA}

The Israeli Journal of Aquaculture (IJA) is an interdisciplinary journal that is dedicated to sharing new research and tested applications of aquaculture

The IJA is devoted to scholarly articles for improved aquaculture practices and related industries

The IJA is a peer-reviewed, open-access, electronic journal

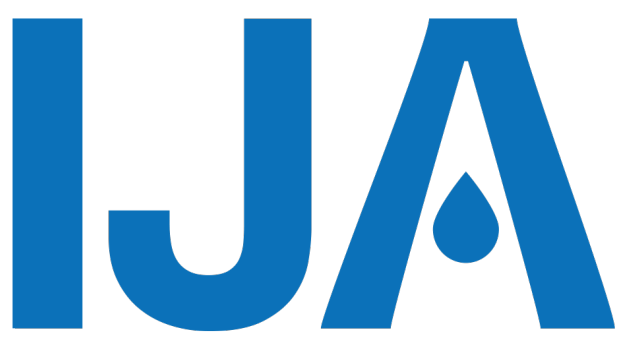

\section{The () Israeli Journal of Aquaculture}

An interdisciplinary online Open Access scientific journal

Published by the

\section{AquacultureHub}

A non-profit organization 501c3

http://www.aquaculturehub.org

in partnership with the

\section{University of Hawaii at Manoa} Library

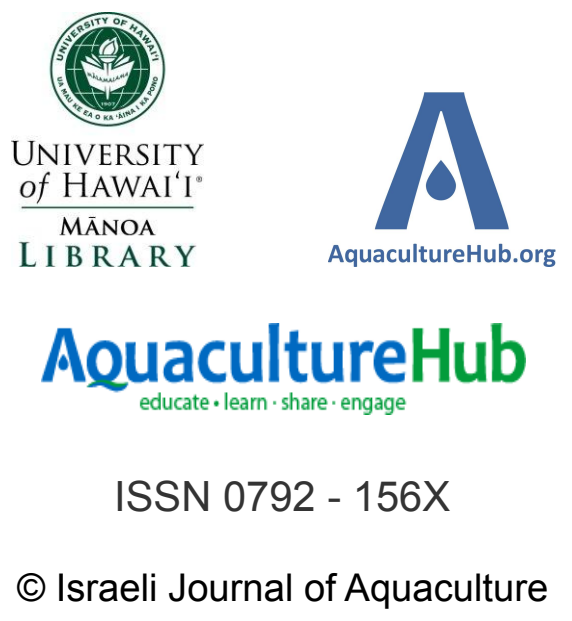



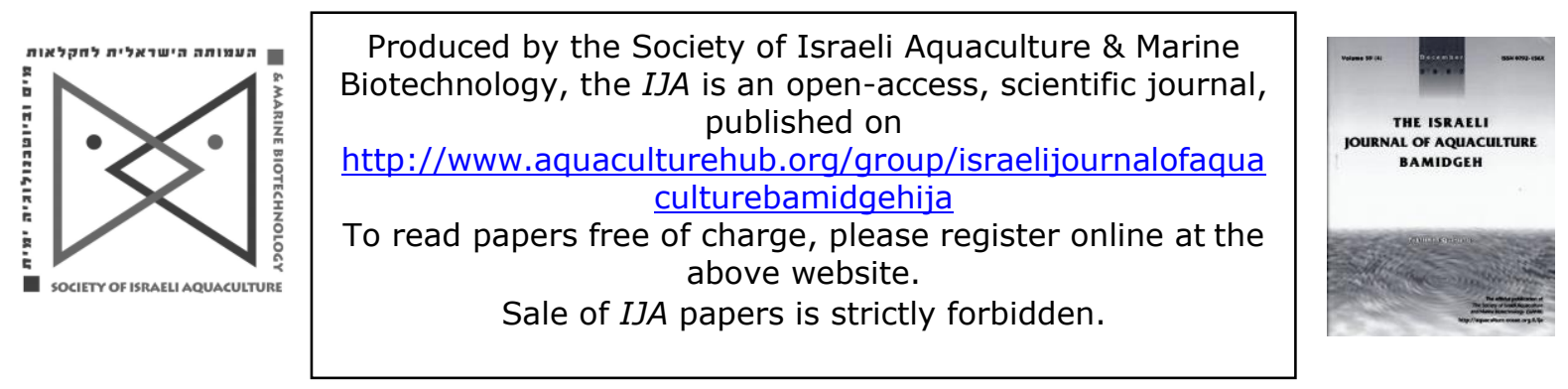

\title{
Advances in Intestinal Mucosal Immunoglobulins of Teleost Fish (Review)
}

\author{
Xia $\mathrm{H}^{1,2}$, ${ }^{\text {, }}$ Yang $\mathrm{P}^{1}$, Liu $\mathrm{L}^{1 *}$, Luo $\mathrm{Y}^{1,2}$, Sun $\mathrm{Y}^{3}$, Wang $\mathbf{W}^{1}$, Chen $\mathrm{N}^{1}$, \\ Zhao $\mathrm{J}^{1}$
}

\author{
${ }^{1}$ Collaborative Innovation Center for Efficient and Health Production of \\ Fisheries in Hunan Province, Hunan University of Arts and Science, Hunan \\ Changde 415000.
}
${ }^{2}$ Co-construction of Provinces and Ministries State Key Experiment on Developmental Biology of Freshwater Fishes, Hunan Normal University, Hunan Changsha 410081.
${ }^{3}$ Hunan Provincial Key Laboratory for Microbial Molecular Biology, Hunan Normal University, Hunan Changsha 410081.

Keywords: intestinal mucosal immune tissue; IgM; IgD; IgZ; teleost fish

\begin{abstract}
Immunoglobulins are globulins with antibody activity or antibody-like structures, and fish were the first vertebrates to develop immunoglobulins. There are three major types of immunoglobulins (IgM, IgD and IgZ) in teleost fish, which play an important role in resisting pathogen infection. For fish, the intestinal tract is not only the place for nutrient digestion but also the largest endocrine organ. The intestinal mucosal barrier system can block pathogenic microorganisms and toxins and is important for immunologic defense. Therefore, the study of the molecular mechanism of fish immunoglobulin in intestinal mucosa is of great significance to the selection of immune prevention strategies for fish diseases. At present, information on the immune system of intestinal mucosa in fish is scarce, and there are still many problems in the function of newly identified antibodies, which need further research. In this study, we have summarized current knowledge about the types, gene structure, biological function, and immune response of immunoglobulins in teleost fish, and intestinal mucosal immune tissue of fish. The scientific questions in this field were also presented according to the progress from the latest research in fish immunology. The aim of present study is to provide a theoretical basis for the study of fish immunology.
\end{abstract}

\footnotetext{
* Corresponding author. Tel.:18890707586, fax:+86 0736-7186016, e-mail:
} xiahu@webmail.hzau.edu.cn, Ilg1818@126.com, yph588@163.com 


\section{Introduction}

Immunoglobulins are globulins with antibody activity or antibody-like structures. They are classified in two groups, namely the secretory and membrane-bound immunoglobulins. Secretory immunoglobulins exist in body fluids and have antibody activity, while membrane-bound immunoglobulins serve as antigen receptors on the surface of B cell membranes, which are related to antigen neutralization. Fish are the first vertebrates to develop immunoglobulins, and adaptive immunity plays an important role in defense against pathogen infection in fish. Meanwhile, due to their special aquatic living environment, there are abundant immunoglobulins in mucosa-associated lymphoid tissues (nose, skin, gill, and intestine) and mucus of fish (Yu et al., 2018; Xu et al., 2016; $\mathrm{Xu}$ et al., 2013; Zhang et al., 2010). These immunoglobulins play an important role in the adaptive immune stage of fish resistance to pathogen infection. Compared with the fish systemic immunity, the mucosal immune antibody was discovered later, and the immune mechanism and its relationship with systemic immunity are not clear. As two independent lymphocyte subsets, the functions of mucosal immune antibody secreting cells still need to be further studied. Furthermore, studying fish immunoglobulins will be helpful to further understand the evolution and occurrence of the immune system.

The gut-associated lymphoid system of teleost fish

For both higher and lower vertebrates such as fish, mucous membranes are more than just a physical barrier that separates them from the environment. The more important role of mucous membranes is to protect the body from external pathogenic microorganisms and stresses, so they also have an active immune function. Due to the special aquatic living environment, the mucosal immunity of fish is more developed. There are a large number of immune active substances and immune cells in the nasal cavity, body surface, gill, and intestinal mucosa of fish. According to the location, the mucosa tissues of fish can be divided into nasal-associated lymphoid tissue, gutassociated lymphoid tissue, skin-associated lymphoid tissue, and gill-associated lymphoid tissue (Yu et al., 2018; Xu et al., 2016; Xu et al., 2013; Zhang et al., 2010).

Teleosts have a complex gut-associated lymphoid system, which is significantly different from that in mammals on morphology and function. In fish, there is no lumped lymphoid tissue and diffuse lymphoid tissue in the intestines, which exist only in birds and mammals (Lazado et al., 2014). However, mucosal epithelium and mucosal lamina exist in fish, and lymphocytes are dispersedly distributed in the digestive tract of fish (Gomez et al., 2013). Among the species studied, there are many types of immune cells in mucosal epithelium and mucosal lamina, including macrophages, granulocytes, lymphocytes, and plasmacytes, which play an important role in intestinal mucosal immune response (Rombout et al., 2014).

The stomach and digestive tract of animals have complex microbial communities, which serve as a beneficial symbiosis with the host mucous membrane environment. In mammals, antibodies play an important role in maintaining intestinal homeostasis. In addition, intestinal microbial community can also regulate the systemic immune response and mucosal immune response. In mammals, intestinal bacteria are mainly coated with IgA, as well as a small amount of IgM and IgG, which prevent their colonization of intestinal mucosal epithelium through immune exclusion (Fritz et al., 2011). Similar to IgA in mammals, the IgT and IgM coats $48 \%$ and $24 \%$ of intestinal bacteria in Oncorhynchus mykiss respectively (Salinas et al., 2011). Studies on Oncorhynchus mykiss have revealed for the first time that immunoglobulins also have immune rejection effects in non-mammalian species. Studies have shown that oral administration of alginate-encapsulated IPNVDNA antigens can increase the number of IgM+ B-cells and IgT+ B-cells, indicating that these two B-cells play an important role in the immune response of fish mucosa (Adelmann et al., 2008). Using monoclonal antibody WCI12 of IgM, researchers examined the intestinal tract of carp by immunohistochemical methods and found that the intestinal intraepithelial lymphocytes (IELs) were IgM positive, and a large number of IgM+ B-cells were actually plasma cells (Koshio, 2016). In the intestinal inflammation model in fish, intraepithelial lymphocytes (IEL) of midgut and hindgut, as well as eosinophil/basophils were involved in the antigen transport and immune response 
(Løkka and Koppang, 2016). However, the immune response mechanism of fish immunoglobulins and the corresponding B lymphocytes in intestinal mucosa during pathogen infection is still unclear and needs to be further studied.

Immunoglobulins of teleost fish

Immunoglobulins are important functional molecules in adaptive immunity of fish and other vertebrates. Mammals have five types of immunoglobulin, namely, IgM, IgA, IgE, IgD, and IgG, while teleost fish have long been thought to have only two types of immunoglobulin, IgM and IgD, and have no antibodies that contribute to mucosal immunity like IgA in mammals (Bao et al., 2010). However, in 2005, a new type of immunoglobulin was found in zebrafish, and was named IgZ (Danilova et al., 2005). Moreover, a new type of immunoglobulin, IgT, was also found in Oncorhynchus mykiss in the same year (Hansen et al., 2005). These two immunoglobulins displayed a high similarity in sequence and clustered into one branch with high support in phylogenetic tree. Therefore, it is thought that IgZ and IgT belong to the same type of immunoglobulin. There are many unsolved questions about the function of these newly discovered antibodies. However, their discovery has prompted new research directions, and that can lead to a better understanding of the differentiation, classification and function of fish antibody subtypes.

IgM

IgM is the first reported immunoglobulin. It plays a crucial role in the first line of defense in the specific immune system (Reddy and Corley, 1999; Klimovich et al., 2008). IgM is mainly produced by B cells in the spleen and lymph nodes and is widely distributed in the blood. Due to its high molecular weight, IgM is also named macroglobulin. Most of the IgM monomers form into tetramers by non-covalent bonds while there are other forms of polymers, which may be a mechanism of antibody diversity in teleosts. In teleosts, IgM is the main immunoglobulin molecule, which is a tetramer composed of covalently linked heavy and light chains (Partula et al., 1996). There are two forms of IgM, namely the membrane binding type and secretory type. The molecular weights of membrane-bound and secretory IgM are different, which is caused by different mRNA splicing patterns (Wilson et al., 1990; Hordvik et al., 1992). sIgM is secreted by B cells and exists in the blood and other body fluids as immune effectors, while mIgM molecules are embedded in $\mathrm{B}$ cell membranes and function as antigen receptors by binding auxiliary molecules to form $B$ cell receptor complexes.

The purification, sequence diversity analysis and phylogenetic tree construction of IgM have been reported in several kinds of fishes, such as Salmo salar (Hatten et al., 2001), Danio rerio (Danilova et al., 2000) and Gadus morhua (Schrøder et al., 1998). IgM plays an important role in humoral immunity, especially in resisting bacterial antigen invasion. IgM expression varies in the different fish species and immune tissues (Schrøder et al., 1998; Koumans-van et al., 1991; Stenvik et al., 2001; Danilova et al., 2002; Grøntvedt et al., 2003). Wang Xinxin et al. found that IgM was mainly expressed in the head kidney, middle kidney, and spleen of Ctenopharyngodon idella, which indicated that these three immune organs were the main sites of IgM expression; meanwhile, quantitative real-time PCR was used for the first time to study the expression of fish IgM gene in different tissues (Wang et al., 2008). IgM is not only involved in systemic immunity against pathogen infection, but also in mucosal immune response. When blunt-snout bream were infected with Aeromonas hydrophila by intraperitoneal injection, the mRNA expression level of the sIgM gene in the kidney, spleen, and liver reached the peak on the 7th day after infection, while the peak was reached on the 14th day in intestinal mucosa (Xia et al., 2014). However, the protein expression pattern of IgM and the distribution, localization and proliferation of the corresponding $B$ lymphocytes (IgM+ B lymphocytes) in intestinal mucosa during Aeromonas hydrophila infection require further investigation.

$\operatorname{Ig} D$

Since identification of IgD was first reported in 1965, it has been the most mysterious antibody (Wu et al., 2014). During the evolutionary process from fish to human, IgD has maintained and played an important role in immune function. Immunoglobulin IgD was 
originally thought to be a newly evolved type of immunoglobulin expressed only in some mammals. In 1997, Wilson et al. found a chimeric gene similar to mammalian IgD $\delta$ chain in catfish, suggesting that IgD should be an ancient immunoglobulin which appeared in the early stage of vertebrate evolution (Wilson et al., 1997). Subsequently, similar genes were identified in Salmo salar (Hordvik et al., 1999), Gadus morhua (Schrøder et al., 1998), Paralichthys olivaceus (Hirono et al., 2003) and Takifugu rubripes (Saha et al., 2004). IgD can be divided into secretory IgD and membrane-bound IgD. Both membrane-bound and secretory IgD were found in Ictalurus punctatus, while, to date, only membrane-bound IgD was found in the other teleost fish (Bengtén et al., 2002). Different from the secretory and membrane-bound IgM in fish, the two forms of IgD in Ictalurus punctatus are not formed by different splicing patterns, but have their own genome sequences, and are produced from direct transcription of their genomic sequences (Bengtén et al., 2006).

Compared with IgD in other vertebrates, fish IgD have two specific features: first, the transcript of fish IgD displays a chimeric structure and shares $\mu 1$ as the first constant region with IgM. The reason may be that there is no suitable cysteine-mediated L-chain binding in fish $\delta 1$ exons, and the binding of $L$ chain can only be achieved through interchain disulfide bond of $\mu 1$ (Wilson et al., 1997). Secondly, fish IgD gene harbors more constant regions, and the exons are generally replicated; the exon copy number in the constant regions of some fish varies significantly. The structural form of IgD in Salmo salar, Ictalurus punctatus, Hippoglossus hippoglossus, and Ctenopharyngodon idella is VDJ- $\mu 1-\delta 1-(\delta 2-\delta 3-\delta 4) 2-\delta 5-\delta 6-\delta 7-T M 1-T M 2$, and there is a replication of " $\delta 2-\delta 3-\delta 4$ " (Solem and Stenvik, 2006; Xiao et al., 2010). The IgD in Takifugu rubripes has a " $\delta 1-\delta 6 "$ copy, which is VDJ- $\mu 1-(\delta 1-\delta 2-\delta 3-\delta 4-\delta 5-\delta 6) 2-\delta 7-T M 1-T M 2$ (Saha et al., 2004). The constant regions " $\delta 5-\delta 6$ " are missing in Oncorhynchus mykiss IgD, and a copy of $\delta 2$ was found after $\delta 4$; the structure of the IgD is VDJ- $\mu 1-\delta 1-\delta 2 a-\delta 3 a-\delta 4 a-\delta 2 b-\delta 7-T M 1-T M 2$ (Hansen et al., 2005). Four constant regions $\delta 3-\delta 6$ are missing in Gadus morhua IgD, which consists of replication of " $\delta 1-\delta 2$ " separated by $\delta y$, and is formed as VDJ- $\mu 1-\delta 1 A-$ $\delta 2 A-\delta y-\delta 1 B-\delta 2 B-\delta 7-T M 1-T M 2$ (Wang et al., 2010). There is no tandem replication in the exons of Paralichthys olivaceus and Siniperca chuatsi IgD (Wang et al., 2010). The cDNA sequence of Megalobrama amblycephala mIgD gene is VDJ- $\mu 1-\delta 1-\delta 2-\delta 3-\delta 4-\delta 5-\delta 6-\delta 7-$ TMs (Xia et al., 2015). There are significant differences in the structure of $\delta$-chain genes between teleost fish and mammals, and the composition of $\delta$-chain genes in different fish is also different. To date, research on IgD in teleost fish has mainly focused on the cloning and sequence analysis of IgD genes, while the immune function of IgD is still unclear.

In mammals, gene knockout mice showed that IgD could replace IgM in the early stage of B cell development, and both IgD and IgM heavy chain defects had a slight effect on mice (Roes and Rajewsky, 1993). Lutz indicated that IgD could replace the function of IgM to a great extent, and the number of mature B cells in peripheral blood of IgD deficient mice was reduced by $30 \%-50 \%$, suggesting that IgD is superior to IgM in function and plays an important role in regulating immune response and immune system balance (Lutz, 1998). However, the immune function of IgD in intestinal mucosal immune system and the immune response to pathogen infection in teleost fishes need to be further studied.

$\operatorname{IgZ} / \operatorname{Ig} T$

IgT/IgZ is the most recently identified type of antibody in vertebrates, that has been found in a variety of fish such as Danio rerio, Cyprinus carpio, Takifugu rubripes, Paralichthys olivaceus, Oncorhynchus mykiss, Ctenopharyngodon idella, Gasterosteidae, Salmo salar, and Megalobrama amblycephala (Hansen et al., 2005; Xiao et al., 2010; Zimmerman et al., 2011; Ryo et al., 2010; Savan et al., 2005; Du et al., 2016; GambónDeza et al., 2010; Tadiso et al., 2011; Xia et al., 2016). Most of these fish have more than one IgT subtypes.

Studies conducted showed that IgZ/IgT plays an important role in the skin, gill and nasal cavity of Oncorhynchus mykiss during parasite infection (Yu et al., 2018; Xu et al., 2016; $\mathrm{Xu}$ et al., 2013). Zhang Yongan et al. found that in the immune response to 
intestinal parasites, the response of Oncorhynchus mykiss IgT to the pathogen was only present in the intestine, while the response of IgM was limited to the serum. Therefore, in the course of parasite infection, IgT is considered to be a class of specific immunoglobulin involved in intestinal mucosal immune response, with functions similar to those of IgA in mammals and birds (Zhang et al., 2010). Megalobrama amblycephala was infected by intraperitoneal injection of Aeromonas hydrophila, and quantitative realtime PCR analysis showed that the sIgZ gene of Megalobrama amblycephala was highly expressed in the intestine; and when Megalobrama amblycephala was infected by Aeromonas hydrophila through immersion infection, the expression of sIgz gene in intestinal mucosa was significantly higher than that in head kidney and spleen (Xia et al., 2016). However, during the Aeromonas hydrophila infection, molecular mechanism of immune response of Megalobrama amblycephala IgZ in intestinal mucosa is still unclear. Whether IgZ is a specific immunoglobulin involved in intestinal mucosal immune responses also remains to be further investigated.

Summary and prospect

In addition to the body surface, intestines define a barrier for preventing direct contact between vertebrate organisms and the external environment. Harmful antigens such as bacteria, viruses, and parasitic eggs will inevitably enter the body together with food. Therefore, the intestinal mucosal immunity plays an important role in maintaining body homeostasis. In fish, the intestinal tract is not only the site of nutrient digestion, but also the largest endocrine organ. The intestinal mucosal barrier system blocks bacteria and other pathogenic microorganisms and toxins and is very important for the immune defense. Therefore, studying the molecular mechanisms of fish immunoglobulins in intestinal mucosa is of great significance for defining immune prevention strategies for fish diseases. To date, the fish immune system is not well understood, and there are still many unsolved problems in the function of newly identified antibodies, which need to be further studied.

\section{Acknowledgements}

The present study was supported by Hunan Natural Science Foundation (Grant No. 2018JJ3373), China Postdoctoral Science Foundation (Grant No. 2019M652748), Hunan Provincial Key Laboratory for Microbial Molecular Biology (Grant No. 2019FW05), the National Natural Science Foundation of China (Grant No. 31572619) and Co-construction of Provinces and Ministries State Key Experiment on Developmental Biology of Freshwater Fishes Open-end Fund project (Grant No.2017KF007)

The authors declare no conflict of interests.

\section{References}

Adelmann M., Köllner B., Bergmann S.M., Fischer U., Lange B., Weitschies W., Enzmann P.J., Fichtner D., 2008. Development of an oral vaccine for immunisation of rainbow trout (Oncorhynchus mykiss) against viral haemorrhagic septicaemia. Vaccine, 26(6):837-844.

Bengtén E., Quiniou S.M., Stuge T.B., Katagiri T., Miller N.W., Clem L.W., Warr G.W., Wilson M., 2002. The IgH locus of the channel catfish, Ictalurus punctatus, contains multiple constant region gene sequences: different genes encode heavy chains of membrane and secreted IgD. J Immunol. 169(5): 2488-2497.

Bengtén E., Quiniou S., Hikima J., Waldbieser G., Warr G.W., Miller N.W., Wilson M., 2006. Structure of the catfish IGH locus: analysis of the region including the single functional IGHM gene. Immunogenetics, 58(10): 831-844.

Bao Y., Wang T., Guo Y., Zhao Z., Li N., Zhao Y., 2010. The immunoglobulin gene loci in the teleost Gasterosteus aculeatus. Fish Shellfish Immunol, 28(1):40-48.

Danilova N., Hohman V.S., Kim E.H., Steiner L.A., 2000. Immunoglobulin variable region diversity in the zebrafish. Immunogenetics, 52: 81-91.

Danilova N., Steiner L.A., 2002. B cells develop in the zebrafish pancreas. PNAS, 99: 13711-13716. 
Danilova N., Bussmann J., Jekosch K., Steiner L.A., 2005. The immunoglobulin heavy-chain locus in zebrafish: identification and expression of a previously unknown isotype, immunoglobulin Z. Nat Immunol, 6:295-302.

Du Y., Tang X., Zhan W., Xing J., Sheng X., 2016. Immunoglobulin Tau Heavy Chain (IgT) in Flounder, Paralichthys olivaceus: Molecular Cloning, Characterization, and Expression Analyses. Int J Mol Sci, 17(9): 1571-1592.

Fritz J.H., Rojas O.L., Simard N., McCarthy D.D., Hapfelmeier S., Rubino S., Robertson S.J., Larijani M., Gosselin J., Ivanov I.I., Martin A., Casellas R., Philpott D.J., Girardin S.E., McCoy K.D., Macpherson A.J., Paige C.J., Gommerman J.L., 2011. Acquisition of a multifunctional IgA+ plasma cell phenotype in the gut. Nature, 481(7380):199-203.

Grøntvedt R.N., Espeild S., 2003. Immunoglobulin producing cells in the spotted wolffish (Anarhichas minor Olfasen): localization in adults and during juvenile development. Dev Comp Immunol, 27: 1 - 10

Gambón-Deza F., Sánchez-Espinel C., Magadán-Mompó S., 2010. Presence of an unique IgT on the IGH locus in three-spined stickleback fish (Gasterosteus aculeatus) and the very recent generation of a repertoire of VH genes. Dev Comp Immunol, 34(2):114122.

Gomez D., Sunyer J.O., 2013. Salinas I. The mucosal imumme system of fish: the evolution of tolerating commensals while fighting pathogens. Fish Shellfish Immunol, 35:1729-1739.

Hordvik I., Voie A.M., Glette J., Male R., Endresen C., 1992. Cloning and sequence analysis of two isotypic IgM heavy chain genes from Atlantic salmon (Salmo salar L.). Eur J Immunol, 22: 2957-2962.

Hordvik I., Thevarajan J., Samdal I., Bastani N., Krossøy B., 1999. Molecular cloning and phylogenetic analysis of the Atlantic salmon Immunoglobulin D gene. Scand J Immunol, 50: 202-210.

Hatten F., Fredriksen Å., Hordvik I., Endresen C., 2001. Presence of IgM in cutaneous mucus, but not in gut mucus of Atlantic salmon, Salmo salar. Serum IgM is rapidly degraded when added to gut mucus. Fish Shellfish Immunol, 11: $257-268$

Hirono I., Nam B.H., Enomoto J., Uchino K., Aoki T., 2003. Cloning and characterization of a cDNA encoding Japanese flounder Paralichthys olivaceus IgD. Fish Shellfish Immunol, 15: 63-70

Hansen J.D., Landis E.D., Phillips R.B., 2005. Discovery of a unique Ig heavy-chain isotype (IgT) in rainbow trout: Implications for a distinctive $B$ cell developmental pathway in teleost fish. Proc Natl Acad Sci USA, 102: 6919-6924.

Koumans-van J.C.E., Wagenaar G.T.M., Rombout J.H.M.W., 1991. Immunocytochemical detection of membrane antigens of carp leucocytes using light and electronic microscropy. Fish Shellfish Immunol, 1: 47 - 57.

Klimovich V.B., Samoilovich M.P., Klimovich B.V., 2008. Problem of J-chain of immunoglobulins. Zh Evol Biokhim Fiziol, 44: 131 - 143.

Koshio S., 2016. Immunotherapies targeting fish mucosal immunity - current knowledge and future perspectives. Front Immunol, 6: 643.

Lutz C., Ledermann B., Kosco-Vilbois M.H., Ochsenbein A.F., Zinkernagel R.M., Köhler G., Brombacher F., 1998. IgD can largely substitute for loss of IgM function in B cells. Nature, 393(6687): 797-801.

Lazado C.C., Caipang C.M., 2014. Mucosal immunity and probiotics in fish. Fish Shellfish Immunol, 39(1):78-89.

Løkka G., Koppang E.0., 2016. Antigen sampling in the fish intestine. Dev Comp Immunol, 64: 138-149.

Partula S., Schwager J., Timmusk S., Pilström L., Charlemagne J., 1996. A second immunoglobulin light chain isotype in the rainbow trout. Immunogenetics, 45: $44-51$.

Roes J., Rajewsky K., 1993. Immunoglobulin D (IgD)-deficient mice reveal an auxiliary receptor function for IgD in antigen-mediated recruitment of B cells. J Exp Med, 177(1): 45-55. 
Reddy P.S., Corley R.B., 1999. The contribution of ER quality control to the biologic functions of secretory IgM. Immunol Today, 20: $582-588$.

Ryo S., Wijdeven R.H., Tyagi A., Hermsen T., Kono T., Karunasagar I., Rombout J.H., Sakai M., Verburg-van Kemenade B.M., Savan R., 2010. Common carp have two subclasses of bonyfish specific antibody IgZ showing differential expression in response to infection. Dev Comp Immunol, 34:1183-1190.

Rombout J.H., Yang G., Kiron V., 2014. Adaptive immune responses at mucosal surfaces of teleost fish. Fish Shellfish Immunol, 40(2): 634-643.

Schrøder M.B., Flano E., Pilstrøm L., 1998. Localization of Ig heavy chain mRNA positive cells in Atlantic cod (Gadus morhua L.) tissues; identified by in situ hybridization. Fish Shellfish Immunol, 8: 565 - 576.

Stenvik J., Jørgensen T.Ø., 2000. Immunoglobulin D (IgD) of Atlantic cod has a unique structure. Immunogenetics, 51: 452-461

Stenvik J., Schorder M.B., Olsen K., Zapata A., Jørgensen T.O., 2001. Expression of immunoglobulin heavy chain transcripts (VH-families,IgM, and IgD) in head kidney and spleen of the Atlantic cod (Gadus morhua L.). Dev Comp Immunol, 25: 291 - 302.

Saha N.R., Suetake H., Kikuchi K., Suzuki Y., 2004. Fugu immunoglobulin D: a highly unusual gene with unprecedented duplications in its constant region. Immunogenetics, 56: 438-447.

Savan R., Aman A., Sato K., Yamaguchi R., Sakai M., 2005. Discovery of a new class of immunoglobulin heavy chain from fugu. Eur J Immunol, 35:3320-3331.

Solem S.T., Stenvik J., 2006. Antibody repertoire development in teleosts--a review with emphasis on salmonids and Gadus morhua L. Dev Comp Immunol, 30(1-2):57-76.

Salinas I., Zhang Y.A., Sunyer J.O., 2011. Mucosal immunoglobulins and B cells of teleost fish. Dev Comp Immunol, 35(12):1346-1365.

Tadiso T.M., Lie K.K., Hordvik I., 2011. Molecular cloning of IgT from Atlantic salmon, and analysis of the relative expression of $\mathrm{T}, \mu$ and $\delta$ in different tissues. Vet Immunol Immunopathol, 139(1):17-26.

Wilson M.R., Marcuz A., Van G.F., Miller N.W., Clem L.W., Middleton D., Warr G.W., 1990. The immunoglobulin $M$ heavy chain constant region gene of the channel catfish Ictalurus punctatus: an unusual mRNA splice pattern produces the membrane form of the molecule. Nucleic Acids Res, 18: 5227-5233

Wilson M., Bengten E., Miller N.W., Clem L.W., Du Pasquier L., Warr G.W., 1997. A novel chimeric Ig heavy chain from a teleost fish shares similarities to IgD. Proc Natl Acad Sci USA, 94: 4593-4597.

Wang X.X., Sun B.J., Chang M.X., 2008. Molecular cloning and expression analysis of immunoglobulin $\mathrm{M}$ heavy chain gene of grass carp (Ctenopharyngodon idellus). J Fish Chin, 32(1): 49-49.

Wang G.L., Luo Y.P., Sun B.J., Xu Z., Xu Q.Q., Nie P., 2010. Cloning and expression of immunoglobulin D heavy chain in mandarin fish, Siniperca chuatsi. J Fishery Sci China, 17(1): 11-20.

Wu Y.J., Huang Q., Chen W.S., 2014. Research progress of IgD and its receptor function and signal. Prog Physiol Sci, 45(1):37-41.

Xiao F.S., Wang Y.P., Yan W., Chang M.X., Yao W.J., Xu Q.Q., Wang X.X., Gao Q., Nie P., 2010. Ig heavy chain genes and their locus in grass carp Ctenopharyngodon idella. Fish Shellfish Immunol, 29(4):594-599.

Xu Z., Parra D., Gómez D., Salinas I., Zhang Y.A., von Gersdorff Jórgensen L., Heinecke R.D., Buchmann K., LaPatra S., Sunyer J.O., 2013. Teleost skin, an ancient mucosal surface that solicits gut-like immune response. Proc Natl Acad Sci U S A, 35: 1729-1739.

Xia H., Wu K., Liu W.J., Gul Y., Wang W.M., Zhang X.Z., 2014. Molecular cloning and expression analysis of immunoglobulin $M$ heavy chain gene of blunt snout bream (Megalobrama amblycephala). Fish Shellfish Immunol, 40(1):129-135.Xia H., Wu K., Liu W., Wang W., Zhang X., 2015. Spatio-temporal expression of blunt snout bream (Megalobrama amblycephala) mIgD and its immune response to Aeromonas hydrophila. Cent Eur J Immunol, 40 (2): 132-141. 
Xu Z., Takizawa F., Parra D., Gómez D., von Gersdorff Jórgensen L., LaPatra S.E., Sunyer J.O., 2016. Mucosal immunoglobulins at respiratory surfaces mark an ancient association that predates the emergence of tetrapods. Nat Commun, 7:10728-10742.

Xia H., Liu W.J., Wu K., Wang W., Zhang X., 2016. sIgZ exhibited maternal transmission in embryonic development and played a prominent role in gill immune response of Megalabrama amblycephala. Fish Shellfish Immunol, 54: 107-117.

Zhang Y.A., Salinas I., Li J., Parra D., Bjork S., Xu Z., LaPatra S.E., Bartholomew J., Sunyer J.O., 2010. IgT, a primitive immunoglobulin class specialized in mucosal immunity. Nat Immunol, 11(9):827-835.

Zimmerman A.M., Moustafa F.M., Romanowski K.E., Steiner L.A., 2011. Zebrafish immunoglobulin IgD: unusual exon usage and quantitative expression profiles with IgM and IgZ/T heavy chain isotypes. Mol Immunol, 48(15-16): 2220-2223.

Yu Y.Y., Kong W., Yin Y.X., Dong F., Huang Z.Y., Yin G.M., Dong S., Salinas I., Zhang Y.A., Xu Z., 2018. Mucosal immunoglobulins protect the olfactory organ of teleost fish against parasitic infection. PLoS Pathog, 14(11): e1007251. 